\title{
Polarization optimization of spin-echo small angle scattering instruments
}

\author{
M. Theo Rekveldt, Chris P. Duif, Wicher H. Kraan, Jeroen Plomp, and Wim G. Bouwman \\ Department of Radiation, Radionuclides \& Reactors, Faculty of Applied Sciences, \\ Delft University of Technology, $2629 \mathrm{JB}$ Delft, The Netherlands
}

(Received 17 November 2007; accepted 17 December 2007; published online 22 January 2008)

\begin{abstract}
The polarization optimization in a small angle scattering spin-echo setup is considered, under the depolarization and phase errors that occur in field transition regions by improper adjustment of inclined magnetized foils as $\pi$-flippers. Various correction procedures are discussed. In these setups with precession fields perpendicular to the beam directions, corrections can be reduced strongly by the use of $\pi$-flippers, and for the remaining errors, correction coils can be constructed. (C) 2008 American Institute of Physics. [DOI: 10.1063/1.2832350]
\end{abstract}

\section{INTRODUCTION}

The neutron spin-echo (SE) technique was introduced more than thirty years ago by Mezei ${ }^{1}$ to measure small energy transfers in materials without the necessity to confine the beam cross section to obtain sufficient resolution. The method is based on the Larmor precessions of neutron polarization in opposite direction in two precession arms, which cancel each other when nothing occurs between the two arms. However, when the beam is scattered by a sample in between the precession arms thereby changing the energy or direction, the echo is disturbed, which could be measured with high accuracy. Small direction changes can be measured by using front ends of the precession regions inclined to the beam direction. For that purpose one needs precession fields perpendicular to the beam direction. The echo change corresponds to very small energy transfers or to small angle changes where a beam with low angular resolution can be used. $^{2-4}$

The first dedicated spin-echo small angle scattering machine was realized in Delft, ${ }^{5}$ using the $\pi$-flipping of magnetized foils as inclined precession front ends between magnetic pole gaps and a second setup with inclined magnetic field poles working with a white neutron beam. See sketch of the first setup in Fig. 1.

At present, a number of SE setups can be used as spinecho small angle scattering (SESANS) setup under the resonance setup with tiltable fields at HMI in Berlin ${ }^{6,7}$ and similar ones at ILL in Grenoble ${ }^{8}$ and FRMII in Munich. ${ }^{9}$ These setups use neutron resonance as a method to create effective large precession angles. Their resonance coils are located in dc field coils, which sharply limit the field regions, in this way avoiding the effects of the field transitions. As an alternative, fields between pole gaps are much larger but sensitive for depolarization and phase inhomogeneities in the field transition regions over the beam cross section. The advantage of using pole gaps is that fields of the order of $0.1 \mathrm{~T}$ can be used, while the resonance setups, using sharply defined field boundaries, cannot use fields higher than about $0.02 \mathrm{~T}$, because of the practical reason that the neutron beam has to pass the material of the field coils.

The analyzed polarization in a SESANS setup measures the so-called SESANS correlation function $G(Z)$, where $Z$ is the spin-echo length, a variable instrumental quantity, which defines the distance over which correlation occurs. ${ }^{4}$ The function $G(Z)$ is the projection along the beam direction of the normalized scattering length density-density correlation perpendicular to the beam. ${ }^{10}$ The technique is becoming important in the study of systems with large correlation lengths far in the micrometer region such as colloids, gratings, cheese spread, and others. ${ }^{11-13}$ To scan correlation lengths up into the micrometer range, relatively large magnetic fields are needed, too strong to use dc coils with sharply limited field regions.

The SESANS setup in Delft (Fig. 1) works with Permalloy films strongly inclined toward the beam direction $\left(5^{\circ}\right)$, inside a pole gap, nearly perpendicular to the main field in the $z x$ plane. The magnetized foils flip the polarization over $\pi$ around the magnetization axis in the precession plane. These films act effectively as the inclined faces of the precession regions. The great advantage of using such foils is the strong inclination achieved which makes very large spinecho lengths scannable with moderate magnetic fields.

For spin-echo devices working at large $Z$ values, one needs very accurate settings of the magnetic fields because $Z$ and also phase errors are proportional to the magnetic field. In addition, the SESANS technique, where strong inclined front and end faces of the precession regions are used, needs extra precautions for the accurate angular settings of these faces.

The main causes of depolarization in setups using magnetic pole gaps are, successively, depolarization and phase inhomogeneities of the polarization in field transitions, and depolarization and phase inhomogeneities by inaccurate settings of the inclination of the Larmor precession faces. A $\pi$-flipper between the pole gaps reduces the effects of depolarization and phase inhomogeneities considerably. ${ }^{14}$ The accurate setting of the inclination is especially important in the setup with magnetized foils.

In this paper we will consider the effects of field transition regions and inaccurate setting of magnetized foils in more detail in various options and discuss methods for measuring and correcting for them, not mechanically, but by 


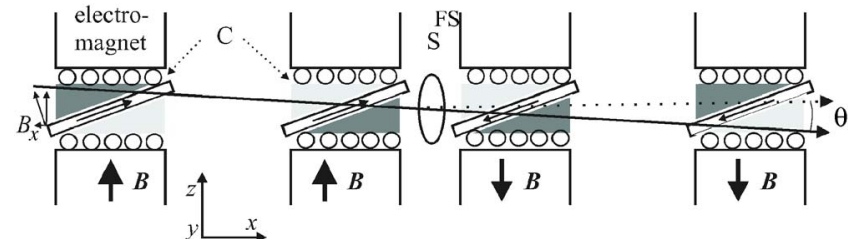

FIG. 1. (Color online) Sketch of the magnetized foil setup in Delft, where the foils are located between the pole gaps of electromagnets. Because of the $\pi$-flip in each foil, the precession phase accumulation reverses as indicated by the shadowed regions between the pole gaps. Except as $\pi$-flipper, the foils in this way also serve as effective inclined faces for the precession regions. Around each foil a coil $(C)$ is wound to generate an $x$ component of the field that causes the local field nearly perpendicular to the foil plane. This also makes the precession inside the foil undergoes a nearly perfect $\pi$-flip. The magnetic induction in the foil is nearly confined to that foil plane. Between the two precession arms, a field stepper (FS) and a sample $S$ are placed.

means of magnetic fields. In the next section we will discuss and characterize the various sources of setup inaccuracies. In Sec. III we discuss possible corrections for field transition region effects in one precession arm using correction coils and we discuss corrections for inaccurate foil settings using the main fields itself and correction coils. In Sec. IV we discuss the depolarization in the whole SE setup and possible corrections in the final SE correlation function. In Sec. V we demonstrate by some experiments the corrections for inaccurate foil settings. We end with conclusions.

\section{DEPOLARIZATION AND PHASE CHANGES IN FIELD TRANSITIONS}

\section{A. Phase inhomogeneity in field transitions for parallel neutron paths}

We describe a field transition at the ends of a pole gap with field $B_{0}$ and an exponential change of the $z$ component of the field in the positive $x$ direction with the $x$ coordinate measured from the edge of the pole gap outwards, in formula,

$$
B_{z}(x)=B_{0} \exp \left(-\frac{x}{\Delta x}\right),
$$

with $\Delta x$ the $1 / e$ length of the transition region that is of the order of the pole gap width. We choose such dependence because the functional dependence is less important and an exponential dependence enables one to derive analytically simple mathematical expressions. The $z$ dependence of the $x$ component of the field is found from Maxwell's law $\operatorname{rot} B=0$, as

$$
B_{x}(x, z)=\frac{d B_{z}(x)}{d x} z=-\frac{B_{z}(x)}{\Delta x} z
$$

with $z$ the distance from the symmetry plane in the pole gap. Because the pole dimensions in the $y$ direction are much larger than the neutron beam width, the $y$ dependence of the field can be neglected. As derived in an earlier paper, ${ }^{14}$ the $z$-dependent term of the field line integral over one transition region is given by

$$
\begin{aligned}
L(z) \equiv L(0)+\Delta L(z) & =\int d x|B(x, z)| \\
& =\int d x\left[B_{z}^{2}(x)+B_{x}^{2}(x, z)\right]^{1 / 2},
\end{aligned}
$$

where the integral is taken over the whole transition region. Using Eq. (2) and $B_{x}(x, z) \ll B_{z}(x)$, the $z$-dependent term of the field line integral is approximated by

$$
\Delta L(z)=\int d x \frac{\left[\frac{d B_{z}(x)}{d x}\right]^{2}}{2 B_{z}(x)}=B_{0} \frac{z^{2}}{2 \Delta x} .
$$

The corresponding extra Larmor phase change in that transition is

$$
\varphi(z)=c \lambda \Delta L(z)=c \lambda B_{0} \frac{z^{2}}{2 \Delta x},
$$

with $c=4.6368 \times 10^{14} \mathrm{~T}^{-1} \mathrm{~m}^{-2}, \lambda$ the wavelength of the neutron beam, and $c \lambda$ the constant which transforms a field path into a Larmor precession phase. For neutron paths not parallel to the $x$ axis, $\varphi(z)$ is only in higher order sensitive for the inclination of the neutron beam with the $x$ axis and, therefore, can be neglected in one transition. The inclination becomes important when the effect of more transitions together is considered. For a beam height $z=0.01 \mathrm{~m}, \Delta x=0.2 \mathrm{~m}, B_{0}$ $=0.1 \mathrm{~T}$, and $\lambda=0.2 \mathrm{~nm}, \varphi(z)$ of a field transition is of the order of $2.5 \mathrm{rad}$. A numerical calculation on a real pole gap field gives no functional difference but only a different constant of the order 1 .

\section{B. Reduction of phase inhomogeneity by $\pi$-flippers}

The phase changes in the field transitions on both sides of the magnets cancel each other largely if a $\pi$-flipper in the magnet is present and in case the divergence of the beam is small. Suppose we have a neutron path $z=a+\theta x$ and pole length $p$ in the $x$ direction. The phase change $\varphi(z)$ of Eq. (5) for the two transitions of one magnetic pole gap reduces to

$$
\varphi(z, \theta)=c \lambda B_{0} \frac{(z+\theta p)^{2}-z^{2}}{2 \Delta x} .
$$

For a beam height $z=0.01 \mathrm{~m}, \Delta x=0.2 \mathrm{~m}, B_{0}=0.1 \mathrm{~T}, \lambda$ $=0.2 \mathrm{~nm}$, and $\theta=0.02, \varphi(z, \theta)$ is about $1 \mathrm{rad}$ for two transitions, at this angle about a factor of 5 less than without $\pi$-flipper. Note the strong angular dependence of the phase $\varphi(z, \theta)$ in this case.

\section{Phase inhomogeneity by beam divergence}

The total field line integral $L_{B}$ is dependent on the type of SE setup we use. Without flipper in the pole gap the field line integral $L_{\mathrm{Bn}}$ is determined by the length $p$ of the magnetic pole gap in the beam direction, the field $B_{0}$ in the pole gap, and the beam divergence $\theta_{d}$. The phase $\varphi_{\mathrm{Bn}}(\theta)$ can be written as

$$
\varphi_{\mathrm{Bn}}(\theta) \equiv c \lambda L_{\mathrm{Bn}}(\theta)=c \lambda \frac{2 B_{0} p}{\cos \theta}=2 c \lambda B_{0} p\left(1+\theta^{2}\right) .
$$


For the foil setup, the field line integral $L_{\mathrm{Bf}}$ over one pole gap is determined by the height $z$ of the beam with respect to the center of magnetized foil flipper, and the corresponding phase can be written as

$$
\varphi_{\mathrm{Bf}}(z, \theta) \equiv c \lambda L_{\mathrm{Bf}}(\theta, z)=2 c \lambda B_{0} \cot \left(\theta_{0}-\theta\right) z .
$$

Here, $\theta_{0}$ is the inclination angle of the foil with the $x$ axis in the $x z$ plane. In the resonance SE setup, the field line integral $L_{\mathrm{Brs}}$ over one precession arm is determined by the distance $L$ between the two magnets in one arm. The corresponding phase is give by

$$
\varphi_{\mathrm{Brs}}(\theta) \equiv c \lambda L_{\mathrm{Brs}}(\theta)=c \lambda \frac{2 B_{0} L}{\cos \theta}=2 c \lambda B_{0} L\left(1+\theta^{2}\right) .
$$

Note the difference in line integral between $L_{\mathrm{Bn}}, L_{\mathrm{Bf}}$, and $L_{\mathrm{Brs}}$. The divergence $\theta$ affects the line integrals in the three cases differently. The effect of flipping in $L_{\mathrm{Brs}}$ with respect to the nonflipping case $L_{\mathrm{Bn}}$ causes the fact that the relevant length in the line integral changes from the length $p$ of the magnet poles to the distance $L$ between the magnets, thus a much larger effect, while the flipping by the inclined magnetized foil causes the fact that there is no net line integral in one SE arm when averaging $L_{\mathrm{Bf}}$ over the diaphragm height $(-z, z)$. To estimate the sensitivity of the first and third line integrals for the beam divergence $\theta_{d}$ take $B_{0}=0.1 \mathrm{~T}$ $p=0.2 \mathrm{~m}, L=1 \mathrm{~m}, \theta_{0}=\pi / 4, \theta_{d}=0.02$, and $z=0.01 \mathrm{~m}$. Then the differences for these options for $\theta=0$ and $\theta=\theta$ are, successively, $\Delta L_{\mathrm{Bn}}=1.6 \times 10^{-5} \mathrm{~T} \mathrm{~m}$ and $\Delta L_{\mathrm{Brs}}=8 \times 10^{-5} \mathrm{~T} \mathrm{~m}$, which at $0.2 \mathrm{~nm}$ neutron wavelength correspond to precession angle differences of tens of radians. The phase difference $\Delta L_{\mathrm{Bf}}=0$ when averaged over some beam height $(-z, z)$ and over all four magnets. However, the $\theta$ dependence in this case in one pole gap plays the essential role in the SESANS effect when combined with the effects in the other four pole gaps, although the average line integral is in echo and is approximately zero. This will be explained in more detail in Sec. IV.

\section{Phase inhomogeneities by inaccurate inclination angle setting}

The SESANS setup, using magnetized foils as inclined precession faces, is extremely sensitive for accurate positioning of the foils. We demonstrate that by a small calculation on the positioning of the foils in the present setup in Delft (Fig. 1) that operates with foil inclination angle of $0.1 \mathrm{rad}$ in a field of $0.1 \mathrm{~T}$. We assume that two basic errors in the mis-settings occur, the angle mis-setting $\Delta \theta$ in orientation in the $x z$ plane and the mis-set angle $\Delta \alpha$ in the $y x$ plane toward another foil in the same SE arm leading to deviations in the precession angles across the beam as function of $y$ and $z$. The phase difference $\Delta \varphi$ as a function of setting errors $\Delta \theta, \Delta \alpha$, and $\Delta B$ causes over a beam height of $z(\mathrm{~cm})$ a precession difference that can easily be calculated for one foil in one pole gap, using Fig. 2 and written in Eq. (10).

$$
\begin{aligned}
\Delta \varphi(y, z)= & 2 c \lambda B \Delta x(z)=-2 c \lambda B\left(z \cot ^{2} \theta_{0} \Delta \theta+y \Delta \alpha\right) \\
& +2 c \lambda z \Delta B \cot \theta_{0}
\end{aligned}
$$

Here, $\Delta x$ is the shift in neutron path when the foil is lifted over a height $z$ that counts positive precession angle instead

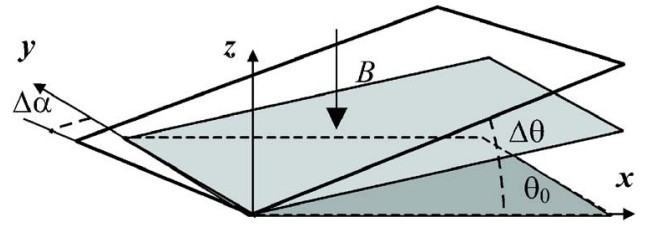

FIG. 2. (Color online) The sketch shows three positions of the foil between the magnetic pole, the first with one leg of the foil along the $y$ axis and the other along the $x$ axis, the second with the foil rotated around the $y$ axis over the angle $\theta_{0}$ to the ideal position, and the third with a mis-setting $\Delta \theta$ around the $y$ axis and a mis-setting $\Delta \alpha$ of a rotation of position 2 around the $z$ axis.

of negative precession when it had passed the foil. Using the numbers above $\lambda=0.2 \mathrm{~nm}, z=\theta_{0} L_{f}$, and foil length $L_{f}$ $=0.12 \mathrm{~m}$, we find $\Delta x=10^{-3} \mathrm{~m}$ and $\Delta \varphi=18 \mathrm{rad}$. If we require that $\Delta \varphi<0.1 \mathrm{rad}$, we have to adjust the foils with an accuracy $\Delta \theta<5 \times 10^{-6}$ or an error of the foil height at one end smaller than $1 \mu \mathrm{m}$.

\section{E. Derivation of equations for foil setting errors in foil setup}

We have to find a procedure to determine the missettings $\Delta \theta$ and $\Delta \alpha$ of the foils, which cause phase inhomogeneities in the $z$ and $y$ directions, respectively. It is clear that the errors manifest themselves most clearly in the measured polarization when the beam passes the foil with the largest cross section, because in that case the polarization has to be averaged over the largest range of precessions $\Delta \varphi$, thus averaging $\cos (\varphi+\Delta \varphi)$. Another way to measure the errors is by scanning $\Delta \varphi$ directly with a pencil beam through the foil.

The second option is used to derive analytical expressions for the mis-settings $\Delta \theta$ and $\Delta \alpha$ of the four foils, expressed in measured phases of the pencil beams. We describe a procedure to correct for the setting errors $\Delta \theta$ and $\Delta \alpha$ in the $y$ and $z$ directions independently. First, we treat the corrections for $\Delta \theta$

The first problem to solve is how to distinguish between the setting errors of the four foils used. Let us define a neutron path through the setup by $z=a+\theta x$ and we assume that the neutron path is not disturbed by a scatterer on his way through the setup. We assume slits at positions $x=0$ and $x$ $=x_{5}$ and the foil positions at $x=x_{1} \cdots x_{4}$, respectively (see Fig. 3 ). For the slits at $x=0$ and $x_{5}$, we give two discrete settings $+d_{z},-d_{z}$ in the $z$ and $+d_{y},-d_{y}$ in the $y$ direction, respectively, which define four independent beams $(--),(-+),(+-)$, $(++)$ by the $z$ slits and four independent beams by the $y$ slits. We will indicate the phases and polarizations measured at these settings by $\varphi_{z}(--) \cdots \varphi_{z}(++)$ and $\varphi_{y}(--) \cdots \varphi_{y}(++)$, respectively. Using Eq. (10) we can write the following four dependent equations for the precession phases of the beams,

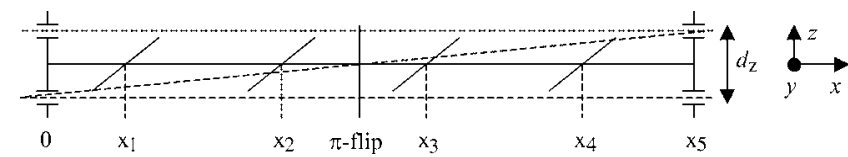

FIG. 3. Sketch of the setup to test the mis-settings of the foils. Two sets of slits with positions $+d_{z}$ and $-d_{z}$ are placed at positions $x=0$ and $x=x_{5}$ along the $z$ direction. Through the slits, three independent neutron paths can be constructed indicated by the broken lines. A similar construction can be made also in the $y$ direction with slit distance $d_{y}$. 
where we realize that, by the $\pi$-flip in the foils themselves and the $\pi$-flipper before $\theta_{3}$, the contribution at $x_{2}$ and $x_{4}$ becomes just negative with respect to the others.

$$
\begin{aligned}
& \varphi_{z}(--)=\varphi_{0}-C_{z} d_{z}\left(\theta_{1}-\theta_{2}-\theta_{3}+\theta_{4}\right), \\
& \varphi_{z}(++)=\varphi_{0}+C_{z} d_{z}\left(\theta_{1}-\theta_{2}-\theta_{3}+\theta_{4}\right), \\
& \varphi_{z}(-+)=\varphi_{z}(--)+C_{z} 2 d_{z}\left(\frac{x_{1}}{x_{5}} \theta_{1}-\frac{x_{2}}{x_{5}} \theta_{2}-\frac{x_{3}}{x_{5}} \theta_{3}+\frac{x_{4}}{x_{5}} \theta_{4}\right), \\
& \varphi_{z}(+-)=\varphi_{z}(++)-C_{z} 2 d_{z}\left(\frac{x_{1}}{x_{5}} \theta_{1}-\frac{x_{2}}{x_{5}} \theta_{2}-\frac{x_{3}}{x_{5}} \theta_{3}+\frac{x_{4}}{x_{5}} \theta_{4}\right),
\end{aligned}
$$

with $C_{z}=2 c \lambda B \cot ^{2} \theta_{0}, \theta_{i} \equiv \Delta \theta_{i}(i=1 \cdots 4)$ of the four foils, and $\varphi_{0}$ an arbitrary phase angle independent of the diaphragm settings. If we can make the four measured precessions angles $\varphi_{z}(--) \cdots \varphi_{z}(++)$ equal by introducing corrections at some foils, then because of the linear behavior of the precessions on the mis-settings [see Eq. (10)] we have achieved a precession angle independent of the transmission angle. From Eqs. (11) only two are independent, one of the first two and one of the last two. We do not need to solve all mis-settings $\theta_{1 \cdots 4}$ of the foils in our SE setup. Our only requirement is that we get a perfect echo over the whole cross section of the beam. That means if we satisfy the equations

$$
\begin{aligned}
& \theta_{1}-\theta_{2}+\theta_{3}-\theta_{4}=0, \\
& x_{1} \theta_{1}-x_{2} \theta_{2}+x_{3} \theta_{3}-x_{4} \theta_{4}=0,
\end{aligned}
$$

then the echo over the whole cross section of the beam is independent of erroneous foil settings $\theta_{1}$ up to $\theta_{4}$. These two conditions can be satisfied by applying only two corrections $C_{1}$ and $C_{2}$ on the foils in the first two magnets 1 and 2 that change $\theta_{i}$ to $\theta_{i}^{\prime}=\theta_{i}+C_{i}(i=1,2)$. This does not mean that we correct for all the mis-settings but making only adjustments to ensure that Eq. (12) is satisfied, i.e.,

$$
\begin{aligned}
& \theta_{1}^{\prime}-\theta_{2}^{\prime}+\theta_{3}-\theta_{4}=0 \\
& x_{1} \theta_{1}^{\prime}-x_{2} \theta_{2}^{\prime}+x_{3} \theta_{3}-x_{4} \theta_{4}=0,
\end{aligned}
$$

or

$$
C_{1}-C_{2}=\frac{\varphi_{z}(++)-\varphi_{z}(--)}{2 C_{z} d_{z}},
$$

and

$$
x_{1} C_{1}-x_{2} C_{2}=\frac{\left[\varphi_{z}(--)-\varphi_{z}(-+)\right] x_{5}}{2 C_{z} d_{z}},
$$

which means that the inclination corrections $C_{1}$ and $C_{2}$ on $\theta_{1}$ and $\theta_{2}$ must be equal to

$$
\begin{aligned}
C_{1}= & \frac{x_{2}}{x_{2}-x_{1}} \frac{\varphi_{z}(++)-\varphi_{z}(--)}{2 C_{z} d_{z}} \\
& -\frac{x_{5}}{x_{2}-x_{1}} \frac{\left[\varphi_{z}(--)-\varphi_{z}(-+)\right]}{2 C_{z} d_{z}},
\end{aligned}
$$

$$
\begin{aligned}
C_{2}= & \frac{x_{1}}{x_{2}-x_{1}} \frac{\varphi_{z}(++)-\varphi_{z}(--)}{2 C_{z} d_{z}} \\
& -\frac{x_{5}}{x_{2}-x_{1}} \frac{\left[\varphi_{z}(--)-\varphi_{z}(-+)\right]}{2 C_{z} d_{z}} .
\end{aligned}
$$

Concluding, we have found the corrections $\left(C_{1}, C_{2}\right)$ on the first two angular settings of the foils that lead to the required phase corrections with the result that the precessions in the three independent neutron paths of Eq. (11) are equal. This procedure has to be applied for slits in the $z$ and $y$ directions to correct the mis-settings $\Delta \theta_{i}$ and $\Delta \alpha_{i}$ successively.

\section{CORRECTIONS}

After having considered the phase inhomogeneities of the different setup parts separately, we will consider now how various corrections can be realized. Some can be realized in each precession arm separately, such as the ones caused by field transitions and some corrections which are dependent on the scattering angle between the precession arms. When we define a neutron path $z(a, \theta)=a+\theta x$, then it appears that the first corrections are described in quadratic terms of $a$ and $\theta$, that can be corrected for by quadratic shaped correction coils. These and other corrections that are dependent on the scattering angle between the precession arms will be considered in their effects on the whole SESANS setup.

\section{A. Corrections for phase inhomogeneities in field transitions}

In case of large beam divergence, it may effectively be advantageous to compensate for the phase changes directly locally by placing correction coils in the transition regions itself. These corrections can be combined with corrections for the path length differences occurring in a divergent beam. Figure 4 shows one precession arm, with a schematic view of two neutron paths in the precession region between two magnets together with the lens shaped correctors $\mathrm{Cor}_{1}, \mathrm{Cor}_{2}$, and $\mathrm{Cor}_{3}$, indicated as $\mathrm{C}_{1}, \mathrm{C}_{2}$, and $\mathrm{C}_{3}$. To compensate for the phase deviations by the field transitions, these phase correctors produce corrections proportional to $z^{2}$, in formula,

$$
\operatorname{Cor}_{i}\left(\alpha_{i}\right)=\alpha_{i} \mathrm{z}^{2}, \quad i=1,2,3 .
$$

We want to adjust the correctors in such a way that the field line integral along any neutron path in the beam has the same value. To do this we have to distinguish the cases with no flippers $(n)$, foil flippers $(f)$, and resonant flippers (rs) in the center of the magnets. Consider along the neutron path $z(a, \theta)$ the corrections in the three phase correctors $\operatorname{Cor}_{i}(i$ $=1,2,3)$, the field transitions $F_{n, f, \mathrm{rs}}=\mathrm{FT} 1(a, \theta)$ $+\cdots \mathrm{FT} 4(a, \theta)$ [see Eqs. (5) and (6)], and the field line integral $L_{\mathrm{Bn}}, L_{\mathrm{Bf}}$, and $L_{\mathrm{Brs}}$ by divergence, introduced in Eqs. (7)-(9). For these cases, the line integral contributions of the field transitions in one precession arm, using Eqs. (4)-(6), can be written as

$$
\begin{aligned}
F_{n}(\theta, L, a, p) & =(\mathrm{FT} 1+\mathrm{FT} 2+\mathrm{FT} 3+\mathrm{FT} 4)(\theta, L, a, p) \\
& =4 \frac{B_{0}}{2 \Delta x}\left[a^{2}+\theta^{2}\left(L^{2}+p^{2}\right)\right],
\end{aligned}
$$




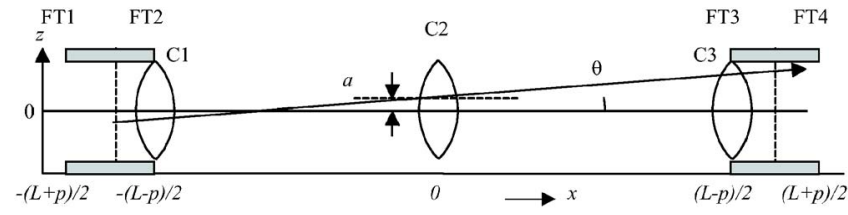

FIG. 4. (Color online) Schematic view of the four field transitions FT1 ‥FT4 and three correction coils in one precession arm of a spin-echo setup with perpendicular fields in the $z$ directions. The shape of the correction coils $\mathrm{C} 1 \cdots \mathrm{C} 3$ symbolizes the quadratic dependence of the field line integral as a function of $z$. Also, the total field line integral is increased by the divergence of the beam by a factor $\left(1+\theta^{2}\right)$, which factor in principle occurs in all components.

$$
\begin{aligned}
\mathrm{F}_{f}(\theta, L, a, p) & =\mathrm{F}_{\mathrm{rs}}(\theta, L, a, p) \\
& =(\mathrm{FT} 1-\mathrm{FT} 2-\mathrm{FT} 3+\mathrm{FT} 4)(\theta, L, a, p) \\
& =4 \frac{B_{0}}{2 \Delta x} \theta^{2} L p,
\end{aligned}
$$

with $L$ and $p$ defined in Fig. 4. The field line integral over one SE arm is found from Eqs. (7)-(9),

$$
\begin{aligned}
& L_{\mathrm{Bn}}(\theta)=2 B_{0} p\left(1+\theta^{2}\right), \\
& L_{\mathrm{Bf}}(a, \theta)=2 B_{0} \cot \left(\theta_{0}\right)(a+\theta L)\left\{1-\theta\left[1+\tan \left(\theta_{0}\right)\right]\right\}, \\
& L_{\mathrm{Brs}}(\theta)=2 B_{0} L\left(1+\theta^{2}\right) .
\end{aligned}
$$

In the second equation [Eq. (18)], $L_{\mathrm{Bf}}(a, \theta)$ contains mixed terms of $a$ and $\theta$. We cannot correct for the term with $a$ with our correctors. However, because this term is an order of magnitude smaller than $L_{\mathrm{Bn}}(\theta)$ we will neglect this term in the following. The term linear in $\theta$ plays the essential role in the SESANS setup and need not be considered in the correction procedure.

The field line contributions of the correction coils are written as

$$
\begin{aligned}
& \operatorname{Cor}_{1}\left(\alpha_{1}, a, \theta\right)=\alpha_{1}\left[a-\frac{\theta(L-p)}{2}\right]^{2}, \\
& \operatorname{Cor}_{2}\left(\alpha_{2}, a, \theta\right)=\alpha_{2} a^{2}, \\
& \operatorname{Cor}_{3}\left(\alpha_{3}, a, \theta\right)=\alpha_{3}\left[a+\frac{\theta(L-p)}{2}\right]^{2} .
\end{aligned}
$$

Here, $\alpha_{\iota}$ are constants of the correction coils which can be adjusted to proper values. Now the $a$ and $\theta$ dependent field path length $\mathbf{L}_{\mathrm{Bn}, \mathrm{f}, \mathrm{rs}}(a, \theta)$ along $z(a, \theta)$ in one precession arm can be written as the sum of the expressions in Eqs. (17)-(19), where the subscripts in $\mathbf{L}$ denote that the expression is valid for the various options with no flipping $(n)$, with a foil flipper $(f)$, and with a resonant flipper (rs) between the magnets.

$$
\begin{aligned}
\mathbf{L}_{\mathrm{Bn}, \mathrm{f}, \mathrm{rs}}(a, \theta)= & L_{\mathrm{Bn}, \mathrm{f}, \mathrm{rs}}(a, \theta)+F_{\mathrm{n}, \mathrm{f}, \mathrm{rs}}(\theta, L, a, p) \\
& +\operatorname{Cor}_{1}\left(\alpha_{1}, a, \theta\right)+\operatorname{Cor}_{2}\left(\alpha_{2}, a, \theta\right) \\
& +\operatorname{Cor}_{3}\left(\alpha_{3}, a, \theta\right) .
\end{aligned}
$$

From Eq. (20) we require that, for proper $\alpha_{1}, \alpha_{2}$, and $\alpha_{3}$, the field path $L_{\mathrm{B}, \mathrm{n}, \pi}(a, \theta)$ should be independent from $a$ and $\theta$, for which we derive the following.

\section{Without flippers}

$$
\begin{aligned}
& \alpha_{1}+\alpha_{2}+\alpha_{3}+\frac{2 B_{0}}{\Delta x}=0, \\
& \alpha_{1}-\alpha_{3}=0, \\
& \left(\alpha_{1}+\alpha_{3}\right)(L-p)^{2}+\frac{2 B_{0}}{\Delta x}\left(L^{2}+p^{2}\right)+2 B_{0} p=0,
\end{aligned}
$$

with solutions

$$
\alpha_{1}=\alpha_{3}=-\frac{B_{0}}{\Delta x} \frac{L^{2}+p^{2}}{(L-p)^{2}}-\frac{B_{0} p}{(L-p)^{2}},
$$

and

$$
\alpha_{2}=\frac{2 B_{0}}{\Delta x} \frac{2 L p}{(L-p)^{2}}+\frac{2 B_{0} p}{(L-p)^{2}} .
$$

\section{With flippers}

$$
\begin{aligned}
& \alpha_{1}+\alpha_{2}+\alpha_{3}=0, \\
& \alpha_{1}-\alpha_{3}=0, \\
& \left(\alpha_{1}+\alpha_{3}\right)(L-p)^{2}+\frac{2 B_{0}}{\Delta x} L p-2 B_{0} L=0,
\end{aligned}
$$

with solutions

$$
\alpha_{1}=\alpha_{3}=-\frac{B_{0}}{\Delta x} \frac{L p}{(L-p)^{2}}+\frac{B_{0} L}{(L-p)^{2}},
$$

and

$$
\alpha_{2}=-2 \alpha_{1} .
$$

The solutions for, $\alpha_{1}, \alpha_{2}$, and $\alpha_{3}$ in both cases show that the corrections are proportional to the field in the magnets as expected. From Eqs. (22) and (24) it follows also that one should make the transition regions $\Delta x$ long enough to be able to correct for the transition with realizable coefficients $\alpha_{1}$, $\alpha_{2}$, and $\alpha_{3}$ of the correctors. Note the difference in size of the correction terms with and without flippers in the magnets. With $B_{0}=0.1 \mathrm{~T}, \Delta x=0.1 \mathrm{~m}$, and neutron path height $a$ $=0.01 \mathrm{~m}$ in Eqs. (18) and (20), we need in the case without flippers a correction of $5 \mathrm{rad}$ at a neutron wavelength of $0.2 \mathrm{~nm}$. With flippers this correction is reduced by a factor $p / L$, which is of the order of 0.1 .

The correction constants of Eq. (24) can also be used for the foil-flipper option to correct for the effects of field transitions but then the second term of $\alpha_{1}$ related to the divergence should be omitted, because the total line integral in the foil option is negligibly small.

Correcting for divergence in the $y$ direction leads to an analogous equation for the constants $\alpha_{1}, \alpha_{2}$, and $\alpha_{3}$ for the coils correcting in the $y$ direction with the difference that the terms with $\Delta x$ do not occur in this case and the total corrections are an order smaller than in the field direction $(z)$.

The technical realization of the correction coils with the property $\operatorname{Cor}_{i}=\alpha_{i} z^{2}$ will be treated in a later section. 


\section{B. Correction for foil angular settings \\ 1. Correction $C_{1}$ and $C_{2}$ of Eq. (15) in the $z$ direction}

For the option to rotate the foil to the correct value, the requirements to the setting of the angle $\theta$ to measure depolarization larger than 0.9 yield an accuracy for the angle of $10^{-5} \mathrm{rad}$. This accuracy is so high that a special goniometer would be needed. Because of lack of space between the magnetic pole shoes we choose for another method.

Instead of correcting the foil angle $\Delta \theta$ in the $z$ direction itself we choose to correct in the Larmor phase angle caused by the incorrect orientation of the foils. The correction for the misalignment in the $z$ direction can simply be done by the field on the foil as one can see immediately from Eq. (10) by considering the difference in Larmor precession over an height $\Delta z$ by a small field change $\Delta B_{z}$ or by a small $\theta_{i}$ change $\Delta \theta_{i}=C_{i}(i=1,2)$, which is given by

$$
\begin{aligned}
& \Delta \varphi=c \lambda \Delta z \cot \theta_{0} \Delta B_{z i}, \\
& \Delta \varphi=c \lambda \Delta z \cot ^{2} \theta_{0} \Delta \theta_{i} B_{z i},
\end{aligned}
$$

from which one derives

$$
\frac{\Delta B_{z i}}{B_{z i}}=C_{i} \cot \theta_{0}
$$

$\Delta B_{z i}$ should change $C_{i} B_{z i} \cot \theta_{0}$ to correct for a misalignment of $\Delta \theta_{i}$ in each foil between the magnetic poles. With $C_{1}$ $\sim \Delta \theta \sim 10^{-4} \mathrm{rad}, \theta_{0}=0.1 \mathrm{rad}$, and a field of $0.1 \mathrm{~T}$, a correction of $10^{-3} B_{z}=10^{-4} \mathrm{~T}$ or $1 \mathrm{G}$ is needed.

If we have chosen to use the field values as a means to correct for the misalignment $\Delta \theta_{i}$, we can, instead of determining the correction parameters $C_{1}$ and $C_{2}$ with narrow diaphragms according to Fig. 3, also directly make a polarization scan with the two relevant magnetic fields $M_{1}$ and $M_{2}$ as parameters. From such a scan one may reveal in addition possible nonlinear deviations of the Larmor precession angle $\varphi$ as a function of height $z$. Figure 9 shows such a scan of the first two magnetic fields, delivering directly the optimal field values.

\section{Correction $C_{1}$ and $C_{2}$ in the $y$ direction}

For corrections in $C_{1}$ and $C_{2}$ in the $y$ direction we have, except the direct rotation of the foil, only one practical way to correct and that is by using a correction coil just close to two of the four magnets in the setup to correct for the deviations in the Larmor precession as a function of $y$.

\section{Correction coils for phase differences in the $y$ and $z$ directions}

To correct for linear precession phase differences in the $y$ direction, a coil can be used as shown in Fig. 5(a), with the cross section of the coil homogeneous in the $z$ direction. In contrast to the correction coil of Fig. 5(a), the correction coil in Fig. 5(b) creates some stray fields around because of the changing cross section in the $z$ direction. However, calculation of the field distribution around the coil shows that these stray fields are low and mainly in the $x$ direction, which makes them not very sensitive to the precession phase angle by the field in the $z$ direction. Figure 6(a) shows the calcu-
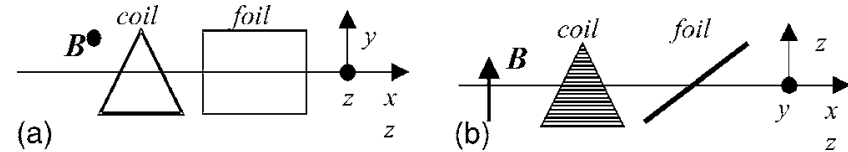

FIG. 5. Correction coils: (a) coil with triangular cross section generating a field in the $z$ direction enables to correct for linear phase differences in field paths shifted along the $y$ direction; (b) coil with changing cross section in the $z$ direction enables to correct for linear phase differences in field paths shifted along the $z$ direction. For each foil a correction coil for $\Delta \alpha$ in the $y x$ plane and another for $\Delta \theta$ in the $z x$ plane have to be applied.

lated field in the $z$ direction as a function of $x$ at two different $z$ values. Figure 6(b) shows the field integrals along the $x$ direction, indicating a sufficient linear behavior.

Similar coils with nonlinear cross sections can be used to correct for nonlinear phase inhomogeneities in $y$ or $z$ that may occur. Such corrections are not possible by the main field as treated in the previous section, because the latter one corrects only linear deviations as that occur in the inclinations of the fields. In practice, we will use for linear misalignment only the correction coils in the $y$ direction, because adjusting the field can perform these corrections in the $z$ direction easier.

\section{DEPOLARIZATION AND PHASE CHANGES IN WHOLE SESANS SETUP WITH TWO PRECESSION ARMS AND $\pi$-FLIPPERS}

Smaller corrections are needed when one considers the whole SESANS setup with two precession arms. When one considers a beam ray in the first arm given by $z(x)=a$ $+\theta x(x<0)$ and in the second arm by $z=a+\left(\theta+\theta_{s}\right) x(x>0)$, where $\theta_{s}$ is the scattering angle by a sample, and assuming that Eq. (5) is valid for the $z$ dependence of the precession angle in the field transition region, the total precession inhomogeneity can be calculated by simply adding the contributions along a neutron path using Eq. (17),

$$
\varphi_{t}\left(\theta_{s}, \theta\right)=\frac{8 c \lambda B_{0} p L}{\Delta x}\left[\left(\theta_{s}+\theta\right)^{2}-\theta^{2}\right] .
$$

Note that this phase only depends on the transmission angles $\theta$ and $\theta+\theta_{s}$ and not on the height where the beam passes the setup. This phase angle averages out over the beam divergence $\left(-\theta_{d}, \theta_{d}\right)$ as

$$
\varphi_{t a}\left(\theta_{s}\right)=\frac{8 c \lambda B_{0} p L}{\Delta x} \theta_{s}^{2},
$$

and for the variation in $\varphi_{t}\left(\theta_{s}, \theta\right)$ with particular $\theta_{s}$ and $\theta$ in the same divergence interval, we find

$$
\Delta \varphi_{t}\left(\theta_{s}, \theta\right) \equiv \sqrt{\varphi_{t}\left(\theta_{s}, \theta\right)^{2}-\varphi_{t a}\left(\theta_{s}\right)^{2}}=\frac{8 c \lambda B_{0} p L}{\Delta x} 2 \theta_{s} \theta .
$$

Written in terms of spin-echo length $Z$ and wave vector transfer $Q$,

$$
\Delta \varphi_{t a}\left(\theta_{s}, \theta\right) \equiv \varepsilon Z Q,
$$

with 


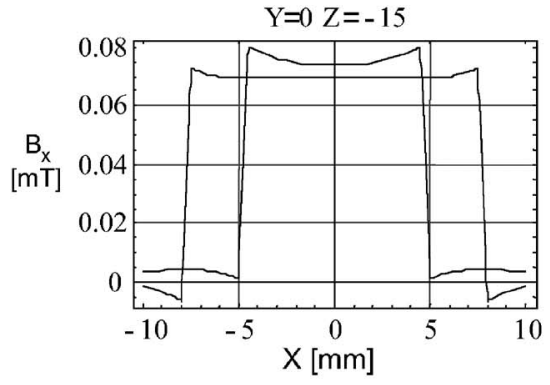

$$
\varepsilon=\frac{16 p \theta}{\Delta x \cot \theta_{0}}, \quad Z=\frac{c \lambda^{2} B L \cot \theta_{0}}{2 \pi},
$$

and

$$
Q=\frac{2 \pi \theta_{s}}{\lambda} .
$$

The depolarization $D\left(\theta_{s}, \theta_{d}\right)$ at every scattering angle $\theta_{s}$ and divergence $\theta_{d}$,

$$
\begin{aligned}
D\left(\theta_{s}, \theta_{d}\right) & \equiv\left\langle\cos \Delta \varphi_{t}\left(\theta_{s}, \theta\right)\right\rangle_{a} \\
& \approx 1-\frac{1}{4 \theta_{d}} \int_{-\theta_{d}}^{\theta_{d}} d \theta\left[\Delta \varphi_{t}\left(\theta_{s}, \theta\right)\right]^{2} \\
& =1-\frac{1}{6}\left(\frac{8 c \lambda B_{0} p L}{\Delta x}\right)^{2}\left(2 \theta_{s} \theta_{d}\right)^{2} \equiv D(\varepsilon) \\
& =1-\varepsilon^{2} \frac{(Z Q)^{2}}{2},
\end{aligned}
$$

with

$$
\varepsilon=\frac{16 p \theta_{d}}{\Delta x \cot \theta_{0} \sqrt{3}},
$$

for $\Delta \varphi_{t}\left(\theta_{s}, \theta_{d}\right) \ll 1$. The error $\varepsilon$ does not depend anymore on the $z$ positions where the beam crosses the field transitions. Although this error is an order of magnitude smaller than the summed errors of the field transition regions, we have no coil system, which could correct for this error as in the case considered for one precession arm separately. However, this error is small for $\theta_{s} \ll \theta_{d}$ and can be corrected for in the data evaluation in the following way.

The Fourier integral for the measured SESANS correlation function $G_{m}(Z)$, including the depolarization of Eq. (31), can be written analogously as in Ref. 4,

$$
G_{m}(Z)=\frac{1}{\sigma k_{0}^{2}} \int d Q_{y} d Q_{z} \frac{d \sigma}{d \Omega}(\mathbf{Q}) \cos \left(Q_{z} Z\right)\left[1-\frac{(\varepsilon Z)^{2}}{2} Q_{z}^{2}\right],
$$

which can be written also as

$$
G_{m}(Z)=G(Z)-\frac{\varepsilon^{2}}{2} Z^{2} \frac{d^{2}}{d Z^{2}} G(Z),
$$

from which $G(Z)$ can be determined by a simple iteration process from the measured $G_{m}(Z)$ when $\varepsilon$ becomes not too large. To get an impression of the depolarization $D(\varepsilon)$, Fig. 7 shows the depolarization as a function of $\varepsilon$ for some given instrumental values.
With the instrumental variables $L=1 \mathrm{~m}, p=\Delta x=0.2 \mathrm{~m}$, $c \lambda=10 \mathrm{~T}^{-1} \mathrm{~m}^{-1}, B_{0}=0.1 \mathrm{~T}$, and $\cot \theta_{0}=10$, the value $\varepsilon$ $\cong \theta_{d} / 2$. So $\varepsilon=0.2$ in the figure corresponds to a large divergence of $20^{\circ}$. If we assume that $Z^{2} d^{2} G(Z) / d Z^{2}$ is of the order 1 , we see that the correction term is negligibly small. For larger values of $\varepsilon$ we can easily correct by a simple iteration procedure.

\section{EXPERIMENTS}

For determining the corrections $C_{1}$ and $C_{2}$ for the foil mis-settings according to Eq. (15) experimentally, one could in principle follow the procedure with small diaphragms as sketched in the derivation of these constants in Sec. II E. However, that procedure has bad statistics because of the narrow slits that must be used. Therefore, we prefer to find the corrections by scanning simply the beam polarization as a function of the first two magnetic fields that would deliver the same results. Figure 8 gives the results for the main fields set at $100 \mathrm{mT}$. The measured polarization is plotted as a function of the deviations $M_{1}$ and $M_{2}$ of the first two magnets from the main fields. From the figure one may observe a clear maximum at some field values of $M_{1}$ and $M_{2}$. Repeating this scan at other field values shows that the determined corrections $M_{1}$ and $M_{2}$ are linear in the main field $B$ as one may expect. Such a scan is simpler to execute than measuring the phases at various diaphragm settings. Therefore, we will use this method also to determine the corrections in the mis-settings $\Delta \alpha_{1}$ and $\Delta \alpha_{2}$ in the $y$ direction. Magnetic fields created in triangular coils with the magnetic field in the $z$ direction, just in front of the magnets $M_{1}$ and $M_{2}$, perform the corrections in the $y$ direction. The triangular shape cre-

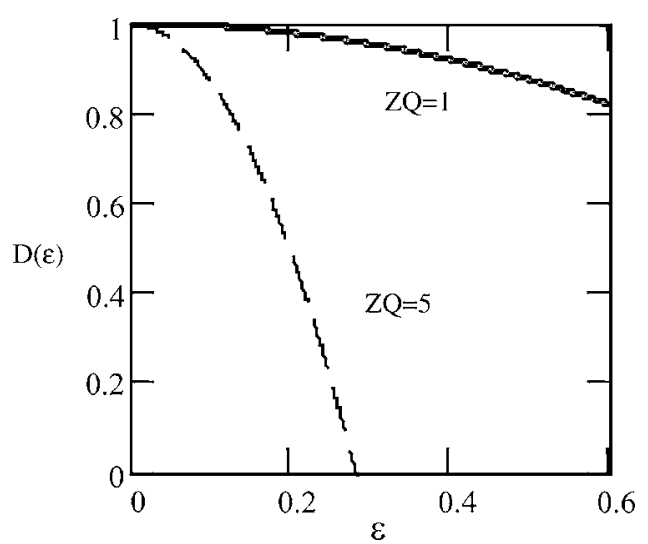

FIG. 7. Calculated depolarization as a function of $\varepsilon$ at two values for the net precession angle $Z Q$ in both precession arms. 


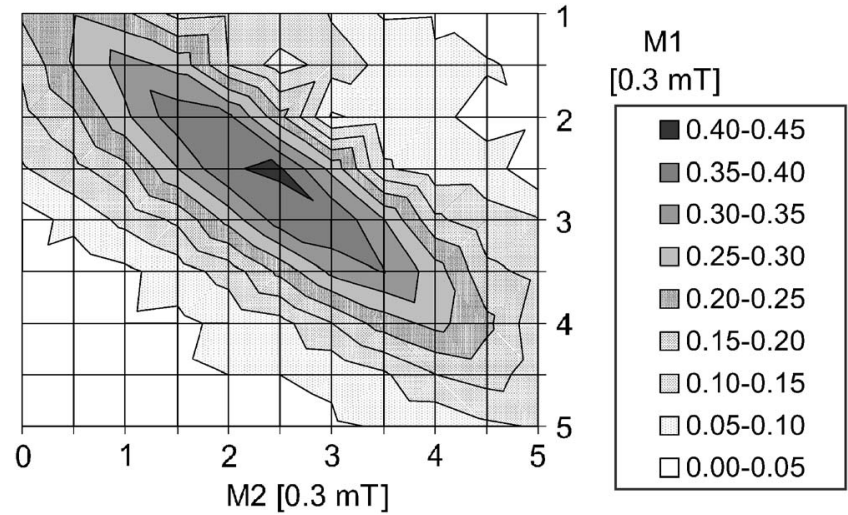

FIG. 8. Polarization scanned as a function of the field deviations $M_{1}$ and $M_{2}$ from the main fields at $100 \mathrm{mT}$ with $M_{1}$ and $M_{2}$ measured in units of $0.3 \mathrm{mT}$.

ates a linear gradient in Larmor precession angle in the $y$ direction. The results of such a scan for corrections in the $y$ direction are shown in Fig. 9, where the polarization is plotted as a function of the currents $y_{1}$ and $y_{2}$ through the correction coils.

The figure also shows a maximum polarization at some correction settings $y_{1}$ and $y_{2}$ of the coils, although the sensitivity of the polarization for these corrections is much weaker than that for the corrections in the $z$ direction. Considering the region of the maximum in more detail, it appears that the maximum is not simple parabolic, but consists of a broad, not well defined, maximum as one would expect from a beam homogeneous in intensity in a field homogeneous over the cross section off the beam. Also, in Fig. 8, one may recognize such irregularities. The homogeneity of the beam intensity and of the magnetic field may be the cause of these deviations, which will be a subject for better beam and field definitions later on.

For the moment our corrections have led already to a polarization improvement, as shown in Fig. 10, where the polarization is plotted as a function of the spin-echo length in the empty beam before and after corrections have been applied.

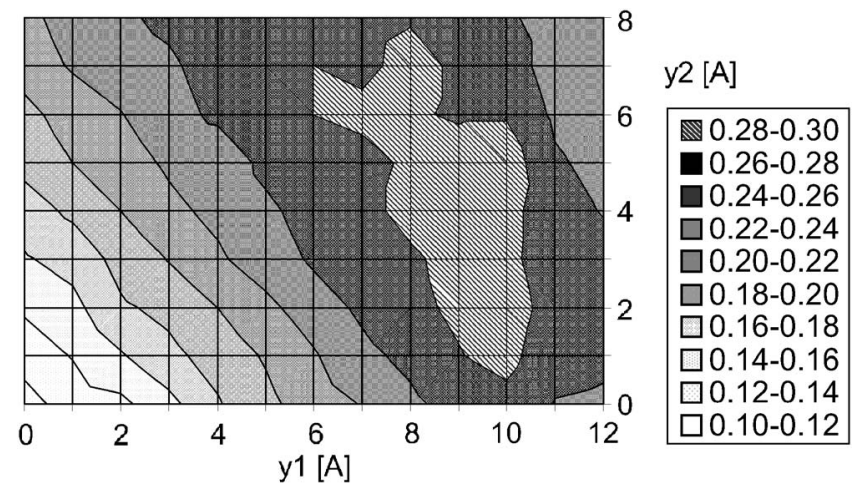

FIG. 9. Polarization measured as a function of the currents $y_{1}$ and $y_{2}$ through the correction coils for the precession corrections in the $y$ direction.

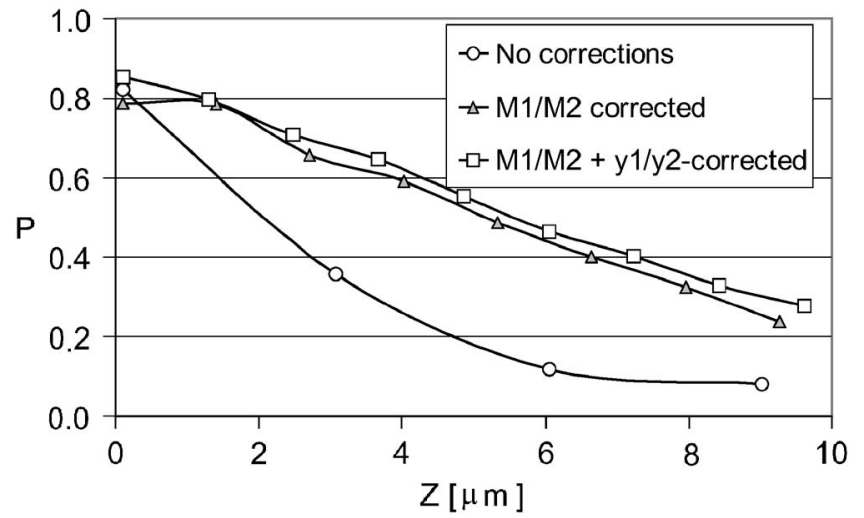

FIG. 10. Polarization as a function of spin-echo length $Z$ before and after correcting for mis-settings of the foils in the $z$ and $y$ and directions.

\section{CONCLUSIONS}

It appears possible to derive analytic expressions for deviations in Larmor precession angles in field transition regions and misadjustment of inclined precession regions. Moreover, it appears possible to construct coils that correct for these deviations occurring in the field direction as well as perpendicular to the field. The effect of $\pi$-flippers in the beam causes a strong reduction in the necessary corrections.

\section{ACKNOWLEDGMENTS}

This research project has been supported in part by the European Commission under the sixth Framework Programme through the Key Action: Strengthening the European Research Area, Research Infrastructures (Contract No. RII3-CT-2003-505925) and by the INTAS foundation (Grant No. INTAS-03-51-6426).

${ }^{1}$ F. Mezei, Lect. Notes Phys. 28, 3 (1980).

${ }^{2}$ R. Pynn, J. Phys. E 11, 1133 (1978).

${ }^{3}$ T. Keller, R. Gähler, H. Kunze, and R. Golub, Neutron News 6, 16 (1995).

${ }^{4}$ M. Th. Rekveldt, W. G. Bouwman, W. H. Kraan, O. Uca, S. V. Grigoriev,

K. Habicht, and T. Keller, Lect. Notes Phys. 601, 87 (2003).

${ }^{5}$ M. T. Rekveldt, J. Plomp, W. G. Bouwman, W. H. Kraan, S. Grigoriev, and M. Blaauw, Rev. Sci. Instrum. 76, 033901 (2005).

${ }^{6}$ T. Keller, R. Golub, F. Mezei, and R. Gähler, Physica B 241-243, 101 (1998)

${ }^{7}$ T. Keller, B. Keimer, K. Habicht, R. Golub, and F. Mezei, Lect. Notes Phys. 601, 74 (2003).

${ }^{8}$ M. Bleuel, F. Demmel, R. Gähler, R. Golub, K. Habicht, T. Keller, S. Klimko, I. Köper, S. Longeville, and S. Prokudaylo, Lect. Notes Phys. 601, 176 (2003)

${ }^{9}$ T. Keller, K. Habicht, H. Klann, M. Ohl, H. Schneider, and B. Keimer, Appl. Phys. A: Mater. Sci. Process. 74, S332 (2002); S. P. Bayrakci, T. Keller, K. Habicht, and B. Keimer, Science 312, 1926 (2006).

${ }^{10}$ T. Krouglov, I. M. de Schepper, W. G. Bouwman, and M. T. Rekveldt, J. Appl. Crystallogr. 36, 117 (2003).

${ }^{11}$ T. Krouglov, W. G. Bouwman, J. Plomp, M. T. Rekveldt, G. J. Vroege, A. V. Petukhov, and D. M. E. Thies-Weesie, J. Appl. Crystallogr. 36, 1417 (2003).

${ }^{12}$ A. Bot, F. P. Duval, and W. G. Bouwman, Food Hydrocolloids 21, 844 (2007).

${ }^{13}$ V. O. de Haan, J. Plomp, W. G. Bouwman, M. Trinker, M. Th. Rekveldt, C. P. Duif, E. Jericha, H. Rauch, and A. A. van Well, J. Appl. Crystallogr. 40, 151 (2007).

${ }^{14}$ M. Th. Rekveldt, W. G. Bouwman, W. H. Kraan, O. Uca, S. V. Grigoriev, and R. Kreuger, Lect. Notes Phys. 601100 (2003). 